\title{
EFFECT OF INDUCED TEMPERATURE FIELD ON DEVELOPMENT OF CURVILINEAR CRACK WITH BONDS BETWEEN THE FACES IN END ZONES
}

\author{
Vagif M. Mirsalimov \\ Azerbaijan Technical University and Institute of Mathematics and Mechanics of NAS of Azerbaijan, Baku, \\ Azerbaijan Republic; e-mail: vagif.mirsalimov@imm.az \\ Azer B. Mustafayev \\ Institute of Mathematics and Mechanics of NAS of Azerbaijan, Baku, Azerbaijan Republic; e-mail: azer_bm@list.ru
}

\begin{abstract}
Temperature changes near the end of a curvilinear cohesive crack and their influence on crack growth are investigated. The problem of local temperature changes consists in a delay or retardation of the cohesive crack growth. The bonds between the curvilinear crack faces in the end zones are modeled by application to the crack surface cohesive forces caused by the presence of bonds. The boundary value problem of equilibrium of the curvilinear crack with interfacial bonds in the end zones under action of external tensile loads, induced temperature field and tractions in the bonds preventing to its opening, is reduced to a system of singular integral equations with a Cauchy-type kernel. From the solution of this equation system, normal and tangential tractions in the bonds are found. Analysis of the limit equilibrium of the crack using the end zone model is performed on the basis of a criterion of bonds limiting stretching and includes: 1) establishment of tractions depending on opening of the crack faces; 2) evaluation of the stress state near the curvilinear crack with taking into account tensile loads, induced temperature field, tractions in the bonds; 3) determination of the critical external tensile loads.
\end{abstract}

Keywords: curvilinear crack with interfacial bonds, thermoelastic stress field, cohesive forces

\section{Introduction}

Creating reliable emergency response systems is a vital issue, especially when we talk about unique installations and safety of people. One of the effective means of crack growth retardation may be temperature and thermoelastic fields (Finkel, 1977; Parton and Morozov, 1985; Potthast and Herrmann, 2000; Fu et al., 2001; Qin et al., 2007; Liu, 2008, 2011a,b, 2014a,b; Georgantzinos and Anifantis, 2014; Liu et al., 2015). In fracture mechanics, the healing problem existing in the crack body is of significant importance (Dimaki et al., 2010). As seen from the results of the papers (Kadiev and Mirsalimov, 2001; Mirsalimov and Kadiev, 2004; Liu, 2008, 2011a,b; Itou, 2014; Liu, 2014a,b; Mirsalimov and Mustafayev, 2015a,b), the influence of the thermal source reduces strain of the stretching plane in the direction perpendicular to the crack, and because of what the stress intensity factor near the crack end lowers. In most of the existing papers, Griffith's model of a crack is used. In the present paper, we use a bridged crack model ([4], Mirsalimov, 2007; Mirsalimov and Mustafayev, 2016).

The crack retardation problem is of scientific and significant practical value as its solution enables one to extend the lifetime and, the main thing, to avoid accidents associated with sudden fracture. Evaluation of efficiency of application of thermal sources in crack growth retardation in thin-walled structural elements is of interest. Fracture of a construction may be prevented by creating a thermal field in the path of crack growth. Creation of thermal fields was justified by their ease of preparation and multilateral nature of influence on the fracture process. 
Technical ease of obtaining in an extended object temperature and thermoelastic field in any size and distribution creates wide opportunities of change of the direction and retardation of crack growth. The experiments (Finkel, 1977) show that by heating the crack path to $70-100^{\circ} \mathrm{C}$, we observe delay and retardation of a crack.

The effect of the temperature field on the retardation of a curvilinear crack with bonds between the faces still has not been investigated. In this connection, studying the influence of the reduced thermal stress field on curvilinear crack propagation in a stretchable plane with regard to the bonds between the faces in the crack end areas is of scientific and practical interest. The goal of the paper is to develop a mathematical model of curvilinear interfacial crack retardation by means of temperature fields.

\section{Formulation of problem}

Let us consider an unbounded elastic plane weakened with a crack of length $2 \ell=b-a$ at the origin of coordinates. In real materials, due to structural and technological factors, crack surfaces have roughnesses and curvings. A fracture mechanics problem on a curvilinear crack in a plane assuming that the crack contour has roughnesses (small deviations from a linear form) is considered. It is assumed that there are areas at which the cohesion forces of the material continuously distributed at the end area of the crack, ect. It is considered that these areas adjoin to the crack tips, their sizes are comparable with the crack length [4]. Models of the crack with end zones were proposed for brittle materials in (Barenblatt, 1961) and for plastic flow state under constant stress in (Leonov and Panasyuk, 1959; Dugdale, 1960). The end zones of the curvilinear crack were simulated by the areas with weakened interparticle bonds in the material.

The model of a crack with interfacial bonds at the end zones may be used in different scales of fracture. Intensive development of crack models with explicit account of nonlinear laws of interaction in conformity to elasto-visco-plastic behavior of materials and various kinds of loading is connected with this fact. Bibliography on this subject may be found in papers of the special issue of Engineering Fracture Mechanics (2003).

When the length of the end zone of the crack is not small compared with the crack length, the methods for evaluating the fracture toughness of the material based on consideration of a crack with a small end zone are not applicable. In these cases, simulation of the stress state at the crack end zone should be carried out with regard to deformational characteristics of the bonds.

The crack faces outside the end zones are free from external loads. At infinity, the strengthened plane is subjected to uniform tension along the ordinate axis by a stress $\sigma_{y}^{\infty}=\sigma_{0}$ (Fig. 1). For retardation of the crack, on the path of its propagation a zone of compressible stresses is formed by means of heating the domain $S$ by a thermal source to temperature $T_{0}$. The following assumptions are accepted:

a) All thermoelastic characteristics of the plate material are temperature independent.

b) The plate material is homogeneous and isotropic.

It is assumed that at the moment $t=0$ an arbitrary domain $S=S_{1}+S_{2}$ on the crack growth path in the plane instantly heats up to a constant temperature $T=T_{0}$. The remaining part of the plate at the initial moment has zero temperature.

For many metallic materials (steels, aluminum alloy and so on) at the temperature change to $300^{\circ} \mathrm{C}-400^{\circ} \mathrm{C}$, the dependence of thermoelastic characteristics weakly changes according to temperature. This fact was experimentally established. Thus, for all structural materials there exists such a temperature range in which the assumption on steadiness of characteristics of the material is correct. It is established on the basis of temperature dependence of the modulus of elasticity. 


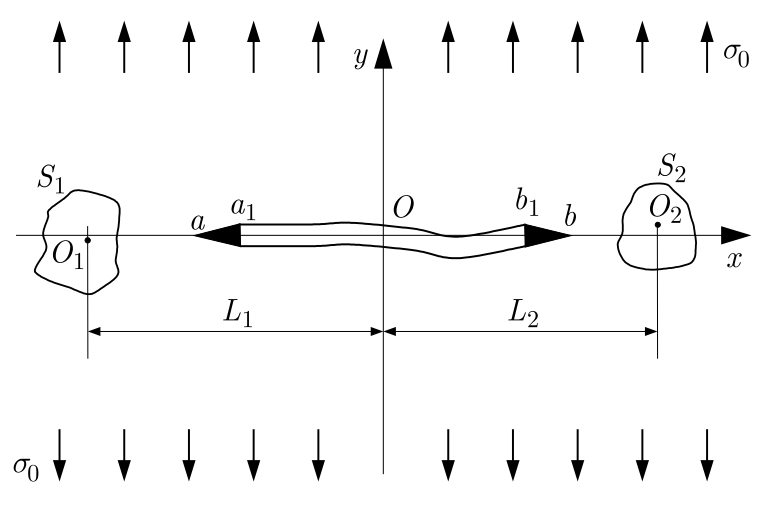

Fig. 1. Computational diagram of the problem

Let us distinguish a part of the crack of length $d_{1}=a_{1}-a$ and $d_{2}=b-b_{1}$ (end zones) adjoining to its tips at which the crack faces interact. The interaction of the crack faces in the end zones is modeled by introducing between the crack faces the bonds (cohesive forces) having the given deformation diagram. The physical nature of such bonds and sizes of the end zones in which the interaction of crack faces is realized, depends on kind of the material. A crack existing in the plane is assumed to be close to a rectilinear form admitting only small deviations of the crack line from the straight line $y=0$. The crack line equation is accepted in the form: $y=f(x)$, $a \leqslant x \leqslant b$. Based on the accepted assumption on the form of the crack line, functions $f(x)$ and $f^{\prime}(x)$ are small quantities.

The end zones are small compared with another part of the plane. Therefore, the end zones may be mentally removed having changed by cuts whose surfaces interact between themselves by some law corresponding to the action of the removed material.

Under the action of external power and thermal loads on the plane, in the bonds connecting the crack faces in the end zones, there will arise in the general case, normal $q_{y}(x)$ and tangential $q_{x y}(x)$ forces. Consequently, to the crack faces in the end zones there will be applied normal and tangential stresses equal to $q_{y}(x)$ and $q_{x y}(x)$, respectively. The quantities of these stresses are not known in advance and they are to be determined.

The boundary conditions of the considered problem have the following form $(y=f(x))$

$$
\sigma_{n}-\mathrm{i} \tau_{n t}= \begin{cases}0 & \text { for } a_{1}<x<b_{1} \\ q_{y}-\mathrm{i} q_{x y} & \text { for } a \leqslant x \leqslant a_{1} \wedge b_{1} \leqslant x \leqslant b\end{cases}
$$

where $n, t$ are natural coordinates. The stress state is represented in the form

$$
\sigma_{x}=\sigma_{x_{0}}+\sigma_{x_{1}} \quad \sigma_{y}=\sigma_{y_{0}}+\sigma_{y_{1}} \quad \tau_{x y}=\tau_{x y_{0}}+\tau_{x y_{1}}
$$

where $\sigma_{x_{0}}, \sigma_{y_{0}}, \tau_{x y_{0}}$ is the solution to the thermoelasticity problem for a crackless plane; $\sigma_{x_{1}}, \sigma_{y_{1}}$, $\tau_{x y_{1}}$ is the stress state of the plane with an interfacial crack on the faces on which the stresses equal in value and opposite in sign, defined by the stress state $\sigma_{x_{0}}, \sigma_{y_{0}}, \tau_{x y_{0}}$ for $y=0$, are additionally applied.

\section{The method of solution of the boundary-value problem}

For determining the stresses $\sigma_{x_{0}}, \sigma_{y_{0}}, \tau_{x y_{0}}$, we solve a thermo-elasticity problem for a solid plane. At first, we find temperature distribution in the plane. For that we solve the following boundary value problem of heat theory

$$
\frac{\partial T}{\partial t}=a_{*} \Delta T \quad T= \begin{cases}T_{0} & \text { for } \quad t=0 \wedge x, y \in S \\ 0 & \text { for } t=0 \wedge x, y \notin S\end{cases}
$$


where $\Delta$ is the Laplace operator; $a_{*}$ is the coefficient of thermal conductivity of the plane material.

For a generalized plane stress state, it is assumed that the plate is thermally insulated on lateral surfaces. When determining the temperature field, for simplifying the problem, we do not take into account a perturbed temperature field caused by the existence of the crack. Let the heated by heat source areas $S_{1}$ and $S_{2}$ from each vertex are arbitrary and simply connected regions with centers $O_{k}\left(L_{k}, c_{k}\right)$ (Fig. 1).

The solution to the thermal conductivity problem has the form

$$
T(x, y, t)=\frac{T_{0}}{4 \pi a_{*} t} \iint_{S_{1}} \exp \left(-\frac{R^{2}}{4 a_{*} t}\right) d \xi d \eta+\iint_{S_{2}} \exp \left(-\frac{R^{2}}{4 a_{*} t}\right) d \xi d \eta
$$

where $R^{2}=(x-\xi)^{2}+(y-\eta)^{2}$.

For the thermoelastic potential of displacement, we find

$$
F(x, y, t)=\frac{(1+\nu) \alpha T_{0}}{4 \pi}\left[\int_{0}^{t} \frac{1}{\tau} \iint_{S} \exp \left(-\frac{R^{2}}{4 a_{*} t}\right) d \xi d \eta-2 \iint_{S} \ln \frac{1}{R} d \xi d \eta\right]
$$

where $S=S_{1}+S_{2} ; \nu$ is the Poisson ratio of the material; $\alpha$ is the coefficient of linear temperature expansion.

The components of the stress tensor are expressed through thermoelastic potential of displacement by the known formulas (Parkus, 1959) and have the form

$$
\begin{aligned}
& \sigma_{x_{0}}=-\mu(1+\nu) \alpha T_{0} \\
& \cdot\left\{1+\frac{1}{\pi} \iint_{S} \frac{1}{R^{4}}\left[(x-\xi)^{2}-(y-\eta)^{2}+2(y-\eta)^{2} \Gamma\left(2, \frac{R^{2}}{4 a_{*} t}\right)-R^{2} \exp \left(-\frac{R^{2}}{4 a_{*} t}\right)\right] d \xi d \eta\right\} \\
& \sigma_{y_{0}}=-\mu(1+\nu) \alpha T_{0} \\
& \quad\left\{1+\frac{1}{\pi} \iint_{S} \frac{1}{R^{4}}\left[-(x-\xi)^{2}+(y-\eta)^{2}+2(x-\xi)^{2} \Gamma\left(2, \frac{R^{2}}{4 a_{*} t}\right)-R^{2} \exp \left(-\frac{R^{2}}{4 a_{*} t}\right)\right] d \xi d \eta\right\} \\
& \tau_{x y_{0}}=-\frac{\mu(1+\nu) \alpha T_{0}}{2 \pi} \iint_{S} \frac{4(x-\xi)(y-\eta)}{\pi} \frac{1}{R^{4}}\left[1-\Gamma\left(2, \frac{R^{2}}{4 a_{*} t}\right)\right] d \xi d \eta \\
& \Gamma(\alpha, x)=\int_{x}^{\infty} \mathrm{e}^{-t} t^{\alpha-1} d t
\end{aligned}
$$

where $\mu$ is the shear modulus of the material.

Boundary conditions (2.1) on the crack with end zones on the basis of (2.2) take the form $(y=f(x))$

$$
\sigma_{n_{1}}-\mathrm{i} \tau_{n_{1}}= \begin{cases}-\left[\sigma_{y_{0}}(x, 0)-\mathrm{i} \tau_{x y_{0}}(x, 0)\right] & \text { for } \quad a_{1}<x<b_{1} \\ q_{y}(x, 0)-\mathrm{i} q_{x y}(x, 0)-\left[\sigma_{y_{0}}(x, 0)-\mathrm{i} \tau_{x y_{0}}(x, 0)\right] & \text { for } \quad a \leqslant x \leqslant a_{1} \wedge b_{1} \leqslant x \leqslant b\end{cases}
$$

Let us consider some arbitrary realization of the curved (with small deviations from the rectilinear form) surface of crack faces.

As the functions $f(x)$ and $f^{\prime}(x)$ are small quantities, the function $f(x)$ may be represented in the form

$$
f(x)=\varepsilon H(x) \quad a \leqslant x \leqslant b
$$


where $\varepsilon$ is a small parameter for which we can accept the greatest height of the bulge of irregularity of the upper crack surface related to the crack length.

The stress and the displacement values and tractions in the bonds are sought in the form of an expansion in the small parameter

$$
\begin{array}{lll}
\sigma_{x_{1}}=\sigma_{x}^{(0)}+\varepsilon \sigma_{x}^{(1)}+\ldots & \sigma_{y_{1}}=\sigma_{y}^{(0)}+\varepsilon \sigma_{y}^{(1)}+\ldots & \tau_{x y_{1}}=\tau_{x y}^{(0)}+\varepsilon \tau_{x y}^{(1)}+\ldots \\
u=u_{0}+\varepsilon u_{1}+\ldots & v=v_{0}+\varepsilon v_{1}+\ldots & \\
q_{y}=q_{y}^{0}+\varepsilon q_{y}^{1}+\ldots & q_{x y}=q_{x y}^{0}+\varepsilon q_{x y}^{1}+\ldots
\end{array}
$$

where the terms with $\varepsilon$ of higher orders are neglected for simplification. Here $\sigma_{x}^{(0)}, \sigma_{y}^{(0)}, \tau_{x y}^{(0)}, u_{0}$, $v_{0}, q_{y}^{0}, q_{x y}^{0}$ and $\sigma_{x}^{(1)}, \sigma_{y}^{(1)}, \tau_{x y}^{(1)}, u_{1}, v_{1}, q_{y}^{1}, q_{x y}^{1}$ are the stresses, displacements and tractions in the bonds of zero and first approximations, respectively.

The values of stresses for $y=f(x)$ can be found by expanding in series the expressions for stresses in the vicinity of $y=0$. Using the procedure of the perturbation method, allowing for previous formulas, we find boundary conditions for $y=0, a \leqslant x \leqslant b$ :

- in the zero approximation $(y=0)$

$$
\sigma_{y}^{(0)}-\mathrm{i} \tau_{x y}^{(0)}= \begin{cases}-\left(\sigma_{y_{0}}-\tau_{x y_{0}}\right) & \text { for } a_{1}<x<b_{1} \\ q_{y}^{0}-\mathrm{i} q_{x y}^{0}-\left(\sigma_{y_{0}}-\tau_{x y_{0}}\right) & \text { for } a^{0} \leqslant x \leqslant a_{1} \wedge b_{1} \leqslant x \leqslant b^{0}\end{cases}
$$

— in the first approximation $(y=0)$

$$
\sigma_{y}^{(1)}-\mathrm{i} \tau_{x y}^{(1)}= \begin{cases}N-\mathrm{i} T & \text { for } a_{1} \leqslant x \leqslant b_{1} \\ q_{y}^{1}-\mathrm{i} q_{x y}^{1}+N-\mathrm{i} T & \text { for } a^{1} \leqslant x \leqslant a_{1} \wedge b_{1} \leqslant x \leqslant b^{1}\end{cases}
$$

where for $y=0$

$$
\begin{aligned}
& N=\tau_{x y}^{(0)} \frac{d H}{d x}-H \frac{\partial \sigma_{y}^{(0)}}{\partial y} \quad T=\left(\sigma_{y}^{(0)}-\sigma_{x}^{(0)}\right) \frac{d H}{d x}-H \frac{\partial \tau_{x y}^{(0)}}{\partial y} \\
& a=a^{0}+\varepsilon a^{1}+\ldots \quad b=b^{0}+\varepsilon b^{1}+\ldots
\end{aligned}
$$

The basic relations of the stated problem should be complemented by the equation connecting the crack faces opening and tractions in the bonds in the end zones of the crack. Without loss of generality, we can represent this equation in the form

$$
\left(v^{+}-v^{-}\right)-\mathrm{i}\left(u^{+}+u^{-}\right)=\Pi_{y}(x, \sigma) q_{y}(x)-\mathrm{i} \Pi_{x}(x, \sigma) q_{x y}(x)
$$

where the functions $\Pi_{y}(x, \sigma)$ and $\Pi_{x}(x, \sigma)$ are effective compliances of the bonds dependent on the tension of the bonds, $\sigma=\sqrt{q_{y}^{2}+q_{x y}^{2}}$ is the modulus of the bonds traction vector; $\left(v^{+}-v^{-}\right)$ are normal and $\left(u^{+}+u^{-}\right)$are tangential components of the opening of the crack faces in the end zones.

At constant values of $\Pi_{y}, \Pi_{x}$ in (3.9), we have a linear law of deformation. In the general case, the deformation law is nonlinear and is given.

The stress strain state $\sigma_{x}^{(0)}, \sigma_{y}^{(0)}, \tau_{x y}^{(0)}$ and $u_{0}, v_{0}$ at an infinite plane and conditions of a plane problem with a cut along the abscissa is described by two piecewise-analytical functions $\Phi(z)$ and $\Omega(z)$ (Muskhelishvili, 2010)

$$
\begin{aligned}
& \sigma_{x}^{(0)}+\sigma_{y}^{(0)}=2\left[\Phi_{0}(z)+\overline{\Phi_{0}(z)}\right] \quad \sigma_{y}^{(0)}-\mathrm{i} \tau_{x y}^{(0)}=\Phi_{0}(z)+\Omega_{0}(\bar{z})+(z-\bar{z}) \overline{\Phi_{0}^{\prime}(z)} \\
& 2 \mu \frac{\partial}{\partial x}\left(u_{o}+\mathrm{i} v_{0}\right)=\kappa \Phi_{0}(z)-\overline{\Phi_{0}(z)}-z \overline{\Phi_{0}^{\prime}(z)}-\overline{\Psi_{0}(z)} \\
& \Omega(z)=\bar{\Phi}(z)+z \overline{\Phi^{\prime}}(z)+\bar{\Psi}(z)
\end{aligned}
$$

where $\kappa=3-4 \nu$ for plane deformation, and $\kappa=(3-\nu) /(1+\nu)$ for a plane stress state. 
For determination of the functions $\Phi_{0}(z)$ and $\Omega_{0}(z)$ on the basis of boundary conditions and the superposition principle, we have a linear conjugation problem (Muskhelishvili, 2010)

$$
\begin{aligned}
& {\left[\Phi_{0}(x)+\Omega_{0}(x)\right]^{+}+\left[\Phi_{0}(x)+\Omega_{0}(x)\right]^{-}=2 p_{0}(x)} \\
& {\left[\Phi_{0}(x)-\Omega_{0}(x)\right]^{+}-\left[\Phi_{0}(x)-\Omega_{0}(x)\right]^{-}=0}
\end{aligned}
$$

where $a \leqslant x \leqslant b, x$ is the affix of the points of the contour of the crack with end zones

$$
p_{0}(x)= \begin{cases}-\left(\sigma_{y_{0}}-\tau_{x y_{0}}\right)-\sigma_{0} & \text { on free faces of the crack } \\ q_{y}^{0}-\mathrm{i} q_{x y}^{0}-\left(\sigma_{y_{0}}-\tau_{x y_{0}}\right)-\sigma_{0} & \text { on faces of the crack end zones }\end{cases}
$$

Since the stresses in the plate are restricted, we should look for the solution to boundary value problem (3.11) in the class of everywhere bounded functions. The sought for solution to the boundary value problem is written in the form

$$
\Phi_{0}(z)=\Omega_{0}(z)=\frac{\sqrt{\left(z-a^{0}\right)\left(z-b^{0}\right)}}{2 \pi \mathrm{i}} \int_{a^{0}}^{b^{0}} \frac{p_{0}}{\sqrt{\left(t-a^{0}\right)\left(t-b^{0}\right)}(t-z)} d t
$$

Moreover, all the following solvability conditions of boundary value problem (3.11) should be fulfilled

$$
\int_{a^{0}}^{b^{0}} \frac{p_{0}(t)}{\sqrt{\left(t-a^{0}\right)\left(b^{0}-t\right)}} d t=0 \quad \int_{a^{0}}^{b^{0}} \frac{t p_{0}(t)}{\sqrt{\left(t-a^{0}\right)\left(b^{0}-t\right)}} g t=0
$$

These relations serve for determination of the unknown parameters $a^{0}$ and $b^{0}$.

For the final determination of the complex potentials $\Phi_{0}(z)$ and $\Omega_{0}(z)$, it is necessary to find the tractions in the bonds $q_{y}^{0}$ and $q_{x y}^{0}$. Using the relation

$$
2 \mu \frac{\partial}{\partial x}\left(u_{0}+\mathrm{i} v_{0}\right)=\kappa \Phi_{0}(z)-\Omega_{0}(\bar{z})-(z-\bar{z}) \overline{\Phi_{0}^{\prime}(z)}
$$

and the boundary values of the functions $\Phi_{0}(z)$ and $\Omega_{0}(z)$ on the segment $a^{0} \leqslant x \leqslant b^{0}$, we get the following equality

$$
\Phi_{0}^{+}(x)-\Phi_{0}^{-}(x)=\frac{2 \mu}{1+\kappa}\left[\frac{\partial}{\partial x}\left(u_{0}^{+}-u_{0}^{-}\right)+\mathrm{i} \frac{\partial}{\partial x}\left(v_{0}^{+}-v_{0}^{-}\right)\right]
$$

Using the Sokhotski-Plemelj formulas (Muskhelishvili, 2010) and taking into account formula (3.12) we find

$$
\Phi_{0}^{+}(x)-\Phi_{0}^{-}(x)=-\frac{\mathrm{i} \sqrt{\left(x-a^{0}\right)\left(b^{0}-x\right)}}{\pi} \int_{a^{0}}^{b^{0}} \frac{p_{0}(t)}{\sqrt{\left(t-a^{0}\right)\left(b^{0}-t\right)}(t-z)} d t
$$

The obtained expression, (3.15), is substituted into the left side of (3.14), and by taking into account relation (3.9) in the zero approximation we get the system of nonlinear integro-differential equations with respect to the unknown functions $q_{y}^{0}$ and $q_{x y}^{0}$

$$
\begin{aligned}
& -\frac{\sqrt{\left(x-a^{0}\right)\left(b^{0}-x\right)}}{\pi} \int_{a^{0}}^{b^{0}} \frac{q_{y}^{0}(t)+f_{y}(t)}{\sqrt{\left(t-a^{0}\right)\left(b^{0}-t\right)}(t-x)} d t=\frac{2 \mu}{1+\kappa} \frac{d}{d x}\left[\Pi_{y}\left(x, \sigma^{0}\right) q_{y}^{0}(x)\right] \\
& -\frac{\sqrt{\left(x-a^{0}\right)\left(b^{0}-x\right)}}{\pi} \int_{a^{0}}^{b^{0}} \frac{q_{x y}^{0}(t)+f_{x y}(t)}{\sqrt{\left(t-a^{0}\right)\left(b^{0}-t\right)}(t-x)} d t=\frac{2 \mu}{1+\kappa} \frac{d}{d x}\left[\Pi_{x}\left(x, \sigma^{0}\right) q_{x y}^{0}(x)\right]
\end{aligned}
$$

Here $f_{y}(t)=-\sigma_{y_{0}}(t)-\sigma_{0}, f_{x y}(t)=-\tau_{x y_{0}}(t)$. 


\section{Numerical solution and analysis}

As expected, the stated problem in the zero approximation is decomposed into two independent problems: Eq. $(3.16)_{1}$ for mode I crack and Eq. (3.16) $)_{2}$ for mode II crack.

Each of equations $(3.16)_{1}$ or $(3.16)_{2}$ is a nonlinear integro-differential equation with a Cauchy-type integral and may be solved only numerically. For their solution, one can use a collocation scheme (Panasyuk et al., 1976; Mirsalimov, 1987; Ladopoulos, 2000, 2013) with an approximation of unknown functions.

Now pass to algebraization of integro-differential equations (3.16). At first, in integro-differential equations (3.16) all integration segments are reduced to one interval $[-1,1]$. For that, we make a change of variables

$$
t=\frac{1}{2}\left(a^{0}+b^{0}\right)-\frac{1}{2}\left(a^{0}-b^{0}\right) \tau \quad x=\frac{1}{2}\left(a^{0}+b^{0}\right)-\frac{1}{2}\left(a^{0}-b^{0}\right) \eta
$$

At such a change of variables, the left side of integro-differential equation $(3.16)_{1}$ admits the following form

$$
-\frac{1}{\pi} \sqrt{1-\eta^{2}}\left[\int_{-1}^{1} \frac{q_{y}^{0}(\tau)}{\sqrt{1-\tau^{2}}(\tau-\eta)} d \tau+\int_{-1}^{1} \frac{f_{y}(\tau)}{\sqrt{1-\tau^{2}}(\tau-\eta)} d \tau\right]
$$

Respectively, for the left side of equation $(3.16)_{2}$ we get

$$
-\frac{1}{\pi} \sqrt{1-\eta^{2}}\left[\int_{-1}^{1} \frac{q_{x y}^{0}(\tau)}{\sqrt{1-\tau^{2}}(\tau-\eta)} d \tau+\int_{-1}^{1} \frac{f_{x y}(\tau)}{\sqrt{1-\tau^{2}}(\tau-\eta)} d \tau\right]
$$

Replace the derivative in the right hand side of equation (3.16) $)_{1}$ for an arbitrary internal $i$-th node by a finite-difference approximation

$$
\frac{d}{d x}\left[\Pi_{y}\left(x, \sigma^{0}\right) q_{y}^{0}(x)\right]_{i}=\frac{\Pi_{y}\left(x_{i+1}, \sigma^{0}\left(x_{i+1}\right)\right) q_{y}^{0}\left(x_{i+1}\right)-\Pi_{y}\left(x_{i-1}, \sigma^{0}\left(x_{i-1}\right)\right) q_{y}^{0}\left(x_{i-1}\right)}{2 \Delta x}
$$

where $\Delta x=\left(b^{0}-a^{0}\right) / M$.

In the similar way we do it with the right hand side of equation $(3.16)_{2}$. Therewith we take into account boundary conditions for $\eta= \pm 1: q_{y}^{0}\left(a^{0}\right)=q_{y}^{0}\left(b^{0}\right)=0, q_{x y}^{0}\left(a^{0}\right)=q_{x y}^{0}\left(b^{0}\right)=0$ (this corresponds to the conditions $v_{0}^{+}\left(a^{0}, 0\right)-v_{0}^{-}\left(a^{0}, 0\right)=0, v_{0}^{+}\left(b^{0}, 0\right)-v_{0}^{-}\left(b^{0}, 0\right)=0, u_{0}^{+}\left(a^{0}, 0\right)-$ $\left.u_{0}^{-}\left(a^{0}, 0\right)=0, u_{0}^{+}\left(b^{0}, 0\right)-u_{0}^{-}\left(b^{0}, 0\right)=0\right)$.

Using the quadrature formula

$$
\frac{1}{2 \pi} \int_{-1}^{1} \frac{g(\tau)}{\sqrt{1-\tau^{2}}(\tau-\eta)} d \tau=-\frac{1}{M \sin \theta} \sum_{k=1}^{M} g_{k} \sum_{m=1}^{M-1} \cos \left(m \theta_{k}\right) \cos (m \theta)
$$

where

$$
\tau=\cos \theta \quad \eta_{m}=\cos \theta_{m} \quad \theta_{m}=\frac{2 m-1}{2 M} \pi \quad(m=1,2, \ldots, M)
$$

all the integrals in (3.16) are replaced by finite sums, and the derivatives in the right hand sides of the equations are replaced by finite-difference approximations.

The above formulae enable one to reduce each integro-differential equation to a finite system of algebraic equations with respect to approximate values of the sought for function, respectively, at the nodal points. As a result, we get 


$$
\begin{aligned}
- & \frac{2}{M}\left[\sum_{\nu=1}^{M} q_{y, \nu}^{0} \sum_{k=1}^{M-1} \sin \left(k \theta_{m}\right) \cos \left(k \theta_{k}\right)+\sum_{\nu=1}^{M} f_{y, \nu} \sum_{k=1}^{M-1} \sin \left(k \theta_{m}\right) \cos \left(k \theta_{k}\right)\right] \\
& =\frac{M(1+\kappa)}{4 \mu\left(b^{0}-a^{0}\right)}\left[\Pi_{y}\left(x_{m+1}, \sigma^{0}\left(x_{m+1}\right)\right) q_{y, m+1}^{0}\left(x_{m+1}\right)-\Pi_{y}\left(x_{m-1}, \sigma^{0}\left(x_{m-1}\right)\right) q_{y, m-1}^{0}\left(x_{m-1}\right)\right] \\
- & \frac{2}{M}\left[\sum_{\nu=1}^{M} q_{x y, \nu}^{0} \sum_{k=1}^{M-1} \sin \left(k \theta_{m}\right) \cos \left(k \theta_{k}\right)+\sum_{\nu=1}^{M} f_{x y, \nu} \sum_{k=1}^{M-1} \sin \left(k \theta_{m}\right) \cos \left(k \theta_{k}\right)\right] \\
& =\frac{M(1+\kappa)}{4 \mu\left(b^{0}-a^{0}\right)}\left[\Pi_{x}\left(x_{m+1}, \sigma^{0}\left(x_{m+1}\right)\right) q_{x y, m+1}^{0}\left(x_{m+1}\right)-\Pi_{x}\left(x_{m-1}, \sigma^{0}\left(x_{m-1}\right)\right) q_{x y, m-1}^{0}\left(x_{m-1}\right)\right]
\end{aligned}
$$

where

$$
\begin{array}{lll}
m=1,2, \ldots, M_{1} & q_{y, \nu}^{0}=q_{y}^{0}\left(\tau_{\nu}\right) & q_{x y, \nu}^{0}=q_{x y}^{0}\left(\tau_{\nu}\right) \\
\sigma_{y_{0}, \nu}=\sigma_{y_{0}}\left(\tau_{\nu}\right) & \tau_{x y_{0}, \nu}=\tau_{x y_{0}}\left(\tau_{\nu}\right) & x_{m+1}=\frac{a^{0}+b^{0}}{2}+\frac{b^{0}-a^{0}}{2} \eta_{m+1}
\end{array}
$$

If we take into account the equality

$$
2 \sum_{k=0}^{M-1} \cos \left(k \theta_{\nu}\right) \sin \left(k \theta_{m}\right)=\cot \frac{\theta_{m} \mp \theta_{\nu}}{2}
$$

the systems will take the following forms

$$
\begin{aligned}
& \sum_{\nu=1}^{M} A_{m \nu}\left(q_{y, \nu}^{0}+f_{y, \nu}\right)=\frac{M(1+\kappa)}{4 \mu\left(b^{0}-a^{0}\right)}\left[\Pi_{y}\left(x_{m+1}, \sigma^{0}\right) q_{y, m+1}^{0}-\Pi_{y}\left(x_{m-1}, \sigma^{0}\right) q_{y, m-1}^{0}\right] \\
& \sum_{\nu=1}^{M} A_{m \nu}\left(q_{x y, \nu}^{0}+f_{x y, \nu}\right)=\frac{M(1+\kappa)}{4 \mu\left(b^{0}-a^{0}\right)}\left[\Pi_{x}\left(x_{m+1}, \sigma^{0}\right) q_{x y, m+1}^{0}-\Pi_{x}\left(x_{m-1}, \sigma^{0}\right) q_{x y, m-1}^{0}\right]
\end{aligned}
$$

where

$$
\begin{array}{lll}
m=1,2, \ldots, M_{1} & q_{y, \nu}=q_{y}^{0}\left(\tau_{\nu}\right) & q_{x y, \nu}=q_{x y}^{0}\left(\tau_{\nu}\right) \\
f_{y, \nu}=f_{y}\left(\tau_{\nu}\right) & f_{x y, \nu}=f_{x y}\left(\tau_{\nu}\right) & A_{m \nu}=-\frac{1}{M} \cot \frac{\theta_{m} \mp \theta_{\nu}}{2}
\end{array}
$$

the upper sign is taken when the number $|m-\nu|$ is odd, the lower sign when it is even.

Now pass to algebraization of the solvability conditions of boundary value problem (3.13). Separating in them the real and imaginary parts, using the change of variables and Gauss's quadrature formula, we get the solvability conditions of the problem in the following form

$$
\begin{aligned}
\sum_{\nu=1}^{M} f_{y}^{*}\left(\cos \theta_{\nu}\right) & =0 & \sum_{\nu=1}^{M} \tau_{\nu} f_{y}^{*}\left(\tau_{\nu}\right) & =0 \\
\sum_{\nu=1}^{M} f_{x y}^{*}\left(\cos \theta_{\nu}\right) & =0 & \sum_{\nu=1}^{M} \tau_{\nu} f_{x y}^{*}\left(\tau_{\nu}\right) & =0
\end{aligned}
$$

where $f_{y}^{*}=q_{y}^{0}+f_{y}, f_{x y}^{*}=q_{x y}^{0}+f_{x y}$.

As a result of algebraization, instead of each integro-differential equation with corresponding additional conditions, we get $M_{1}+2\left(M_{1}\right.$ is the number of nodal points belonging to the crack end zones) algebraic equations to determine stresses at nodal points and the end zone sizes. Even in the special case of linear elastic bonds, the obtained system of equations becomes nonlinear because of the unknown size of the end zone. In this connection, for solving the obtained systems, in the case of linear bonds the method of successive approximations has been used.

In the case of a deformation law of nonlinear bonds, to define tractions in the end zones it is advisable to use an iterative scheme similar to the method of elastic solutions (Il'yushin, 2003). 
It is accepted that the law of deformation of interparticle bonds (cohesive forces) is linear for $V=\sqrt{\left(v_{0}^{+}-v_{0}^{-}\right)^{2}+\left(u_{0}^{+}-u_{0}^{-}\right)^{2}} \leqslant V_{*}$. The first step of iterative calculations is solving the system of equations (4.2) and (4.3) for linear-elastic interparticle bonds. The subsequent iterations are fulfilled only in the case when the relation $V(x)>V_{*}$ holds on a part of the end zone. For such iterations, the system of equations is solved at each approximation for quasi-brittle bonds with effective compliance variable along the crack end zone and dependent on the size of modulus of the fraction vector obtained in the previous step of calculation. Effective compliance analysis is conducted through the definition of the secant modulus in elasticity parameters. The successive approximations process ends as the forces along the end zone, obtained at two successive iterations, differ a little from each other.

The nonlinear part of the strain curve of interparticle bonds is represented in the form of a bilinear dependence whose ascending portion corresponds to elastic deformation of the bonds $\left(0<V(x)<V_{*}\right)$ with their maximum tension. For $V(x)>V_{*}$, the deformation law is described by a non-linear dependence determined by two points $\left(V_{*}, \sigma_{*}\right)$ and $\left(\delta_{c}, \sigma_{c}\right)$. And for $\sigma_{c} \geqslant \sigma_{*}$, we have a descending linear dependence (linear hardening corresponding to elasticplastic deformation of bonds). Here $\sigma_{*}$ is the maximum elastic stress in the bonds, $\delta_{c}$ is the characteristic of fracture toughness of the material determined experimentally. In numerical calculations, it has been assumed $M=30$, which corresponds to partition of the integration interval into 30 Chebyshev nodal points.

After finding stress components in the zero approximation, we find the functions $N$ and $T$ from formulas (3.8). The sequence of solution of problem (3.7) in the first approximation is similar to the solution of the problem in the zero approximation. The solution of the problem on the definition of piecewise-analytic functions $\Phi_{1}(z)$ and $\Omega_{1}(z)$ is of the form

$$
\Phi_{1}(z)=\Omega_{1}(z)=\frac{\sqrt{\left(z-a^{1}\right)\left(z-b^{1}\right)}}{2 \pi \mathrm{i}} \int_{a^{1}}^{b^{1}} \frac{p_{1}(t)}{\sqrt{\left(t-a^{1}\right)\left(t-b^{1}\right)}(t-z)} d t
$$

where

$$
p_{1}(t)= \begin{cases}N-\mathrm{i} T & \text { on free crack faces } \\ q_{y}^{1}-\mathrm{i} q_{x y}^{1}+N-\mathrm{i} T & \text { on faces of the crack end zones }\end{cases}
$$

Moreover, all the following solvability conditions of the boundary value problem should be fulfilled

$$
\int_{a^{1}}^{b^{1}} \frac{p_{1}(t)}{\sqrt{\left(t-a^{1}\right)\left(b^{1}-t\right)}} d t=0 \quad \int_{a^{1}}^{b^{1}} \frac{t p_{1}(t)}{\sqrt{\left(t-a^{1}\right)\left(b^{1}-t\right)}} d t=0
$$

These relations serve for determination of the unknown parameters $a^{1}$ and $b^{1}$.

Using the formula and boundary values of the functions $\Phi_{1}(z), \Omega_{1}(z)$ on the segment $a^{1} \leqslant x \leqslant b^{1}$, we find the equality

$$
\Phi_{1}^{+}(x)-\Phi_{1}^{-}(x)=\frac{2 \mu}{1+\kappa}\left[\frac{\partial}{\partial x}\left(u_{1}^{+}-u_{1}^{-}\right)+\mathrm{i} \frac{\partial}{\partial x}\left(v_{1}^{+}-v_{1}^{-}\right)\right]
$$

Using the Sokhotski-Plemelj (Muskhelishvili, 2010) and taking into account formula (4.4), we find

$$
\Phi_{1}^{+}(x)-\Phi_{1}^{-}(x)=-\frac{\mathrm{i}}{\pi} \sqrt{\left(x-a^{1}\right)\left(b^{1}-x\right)} \int_{a^{1}}^{b^{1}} \frac{p_{1}(t)}{\sqrt{\left(t-a^{1}\right)\left(b^{1}-t\right)}(t-x)} d t
$$


Substituting obtained expression (4.7) into the left part of equation (4.6) and taking into account relation (3.9) for the first approximation, after some transformations we get a system of nonlinear integro-differential equations with respect to the unknown functions $q_{y}^{1}$ and $q_{x y}^{1}$

$$
\begin{aligned}
& -\frac{\sqrt{\left(x-a^{1}\right)\left(b^{1}-x\right)}}{\pi} \int_{a^{1}}^{b^{1}} \frac{q_{y}^{1}(t)+N(t)}{\sqrt{\left(t-a^{1}\right)\left(b^{1}-t\right)}(t-x)} d t=\frac{2 \mu}{1+\kappa} \frac{d}{d x}\left[\Pi_{y}\left(x, \sigma^{1}\right) q_{y}^{1}(x)\right] \\
& -\frac{\sqrt{\left(x-a^{1}\right)\left(b^{1}-x\right)}}{\pi} \int_{a^{1}}^{b^{1}} \frac{q_{x y}^{1}(t)+T(t)}{\sqrt{\left(t-a^{1}\right)\left(b^{1}-t\right)}(t-x)} d t=\frac{2 \mu}{1+\kappa} \frac{d}{d x}\left[\Pi_{x}\left(x, \sigma^{1}\right) q_{x y}^{1}(x)\right]
\end{aligned}
$$

where $\sigma^{1}=\sqrt{\left(q_{y}^{1}\right)^{2}+\left(q_{x y}^{1}\right)^{2}}$.

Similarly, in the first approximation, in obtaining the algebraic systems all integration intervals are reduced to one interval $[-1,1]$. Then, using the quadrature formulas of the Gauss type the integrals are replaced by finite sums. As a result we get the zero approximation while obtaining algebraic systems all integration intervals are reduced to one interval $[-1,1]$. Then, the integrals are replaced by finite sums by means of Gauss-type quadrature formulas. As a result, we get

$$
\begin{aligned}
& \sum_{\nu=1}^{M} A_{m \nu}\left[q_{y, \nu}^{1}+N_{\nu}\right]=\frac{\mu M}{(1+\kappa)\left(b^{1}-a^{1}\right)}\left[\Pi_{y}\left(x_{m+1}^{1}, \sigma^{1}\right) q_{y, m+1}^{1}-\Pi_{y}\left(x_{m-1}^{1}, \sigma^{1}\right) q_{y, m-1}^{1}\right] \\
& \sum_{\nu=1}^{M} A_{m \nu}\left[q_{x y, \nu}^{1}+T_{\nu}\right]=\frac{\mu M}{(1+\kappa)\left(b^{1}-a^{1}\right)}\left[\Pi_{x}\left(x_{m+1}^{1}, \sigma^{1}\right) q_{x y, m+1}^{1}-\Pi_{x}\left(x_{m-1}^{1}, \sigma^{1}\right) q_{x y, m-1}^{1}\right]
\end{aligned}
$$

where

$$
\begin{array}{lll}
m=1,2, \ldots, M_{1} & x_{m+1}^{1}=\frac{1}{2}\left(a^{1}+b^{1}\right)-\frac{1}{2}\left(a^{1}-b^{1}\right) \eta_{m+1} & q_{y, \nu}^{1}=q_{y}^{1}\left(\tau_{\nu}\right) \\
q_{x y, \nu}^{1}=q_{x y}^{1}\left(\tau_{\nu}\right) & N_{\nu}=N\left(\tau_{\nu}\right) & T_{\nu}=T\left(\tau_{\nu}\right)
\end{array}
$$

As a result of algebraization of the boundary problem solvability conditions (4.5), we obtain

$$
\begin{array}{lll}
\sum_{\nu=1}^{M} f_{y}^{* 1}\left(\tau_{\nu}\right)=0 & \sum_{\nu=1}^{M} \tau_{\nu} f_{y}^{* 1}\left(\tau_{\nu}\right)=0 \\
\sum_{\nu=1}^{M} f_{x y}^{* 1}\left(\tau_{\nu}\right)=0 & \sum_{\nu=1}^{M} \tau_{\nu} f_{x y}^{* 1}\left(\tau_{\nu}\right)=0
\end{array}
$$

where $f_{y}^{* 1}=q_{y}^{1}+N, f_{x y}^{* 1}=q_{x y}^{1}+T$.

As a result of algebraization, as in the zero approximation, instead of each integro-differential equation we get a system of $M_{1}+2$ algebraic equations for determining stresses at nodal points of the crack end zones and end zones sizes.

A solving algorithm for algebraic systems (4.9) and (4.10) is similar to the solution of the systems for the zero approximation. The opening of the crack in the end zones may be determined from the relations

$$
\begin{aligned}
& v^{+}(x, 0)-v^{-}(x, 0)=\Pi_{y}(x, \sigma) q_{y}(x) \\
& u^{+}(x, 0)-u^{-}(x, 0)=\Pi_{x}(x, \sigma) q_{x y}(x) \\
& a \leqslant x \leqslant a_{1} \quad b_{1} \leqslant x \leqslant b
\end{aligned}
$$

Calculations show that for the linear law of deformation of bonds, tractions in the bonds have always maximal values at the edge of the end zone. The similar picture is observed for 
the quantities of crack openings. Opening of a crack at the edge of end zone has maximum for linear and nonlinear laws of deformation, and with an increasing compliance of the bonds. To determine the limit state at which the crack growth occurs, we use the critical condition

$$
\left|\left(v^{+}-v^{-}\right)-\mathrm{i}\left(u^{+}+u^{-}\right)\right|=\delta_{c}
$$

The joint solution of the obtained equations and condition (4.12) enable determination of the critical value of external loads, forces in the bonds and the size of the end zone for the limit equilibrium state under the given characteristics of bonds.

The function $H(x)$ describing roughness of the crack surface are considered as a determinate set of the rough surface of the profile contour and also as a stationary random function. In this case, the random function $H(x)$ is given by the canonical expansion

$$
H(\theta)=\sum_{k=-\infty}^{\infty} v_{k} \varepsilon^{\mathrm{i} k \theta}
$$

where $v_{k}$ are independent zero random values of the mathematical expectation and dispersions $D_{k}$.

Calculations show that the heated zone promotes flow of plastic deformations in the bonds.

In Fig. 2a, plots of distribution of normal forces $q_{y}$ in the bonds of the crack end zones are depicted for the following values of free parameters $t_{*}=4 a_{*} t / L_{1}^{2}=10 ; c_{1} / L_{1}=-0.2$; $c_{2} / L_{2}=0.1=0.1 ; \nu=0.3 ; L_{1}=L_{2}=L ; M=30 ; E=1.8 \cdot 10^{5} \mathrm{MPa} ; V_{*}=10^{-6} \mathrm{~m} ;$ $\sigma_{*}=75 \mathrm{MPa} ; \sigma_{c} / \sigma_{*}=2 ; \delta_{c}=2.5 \cdot 10^{-6} \mathrm{~m} ; C=2 \cdot 10^{-7} \mathrm{~m} / \mathrm{MPa}(C$ is the effective compliance of the bonds). There, curves 1 correspond to the bilinear law of strains of the bonds, and curves 2 correspond to the linear law of strains. In the computations, we used the dimensionless coordinates $x=(a+b) / 2+(b-a) x^{\prime} / 2$.

The compliance of the bonds in the normal and tangential directions is assumed to be equal.

Graphs of the distribution of tangential forces $q_{x y}$ in the bonds of the crack end zones are shown in Fig. $2 b$.

(a)

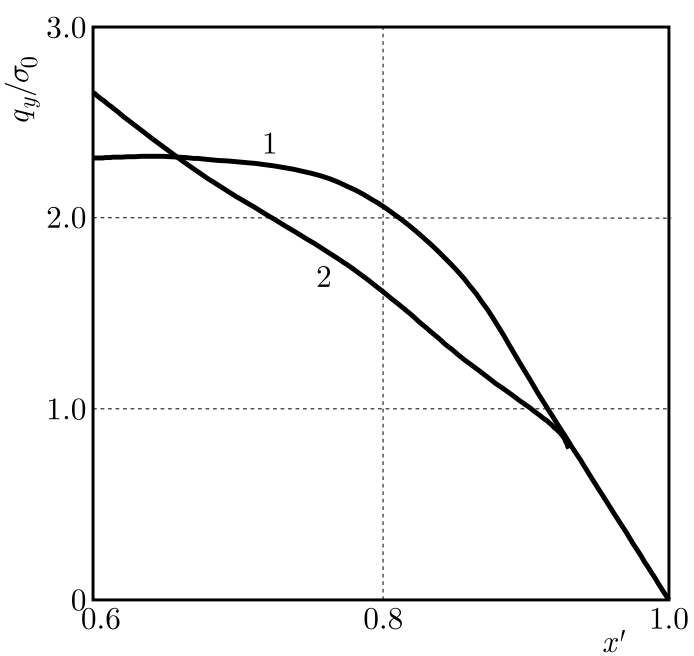

(b)

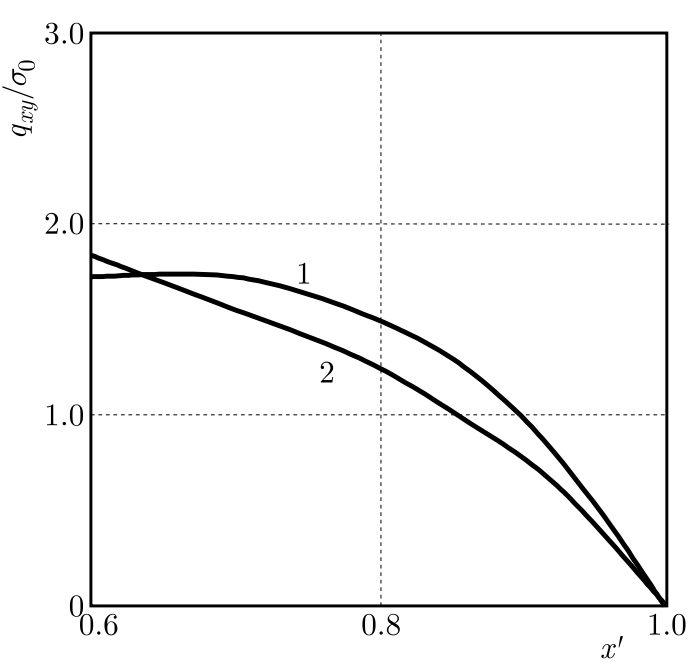

Fig. 2. Distribution of normal forces $q_{y} / \sigma_{0}$ (a) and tangential forces $q_{x y} / \sigma_{0}$ (b) in the bonds of the crack end zones

Graphs of the length of the crack end zone $\left(b-b_{1}\right) /(b-a)$ for the plate against the dimensionless value of the tensile stress $\sigma_{0} / \sigma_{*}$ are shown in Fig. 3 for different crack lengths $\ell_{*}=\left(b_{1}-a_{1}\right) /(b-a)=0.5,0.7$.

The dependence of the critical load $\sigma_{0} / \sigma_{*}$ on the dimensionless length of the crack $(b-a) / R$ is shown in Fig. 4. There $R$ is the typical linear size of the plate. 


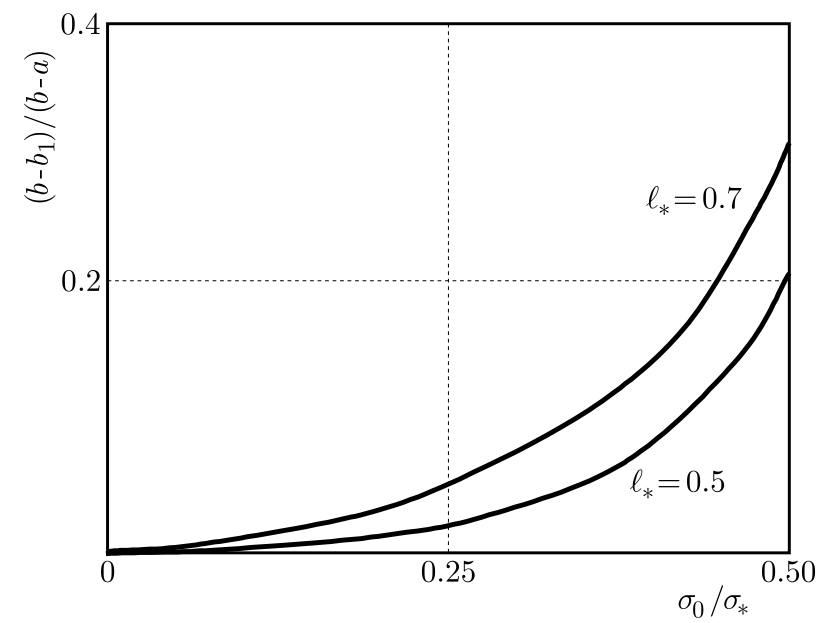

Fig. 3. Dependency of the length of the end zone $\left(b-b_{1}\right) /(b-a)$ on the dimensionless value $\sigma_{0} / \sigma_{*}$ for different crack lengths

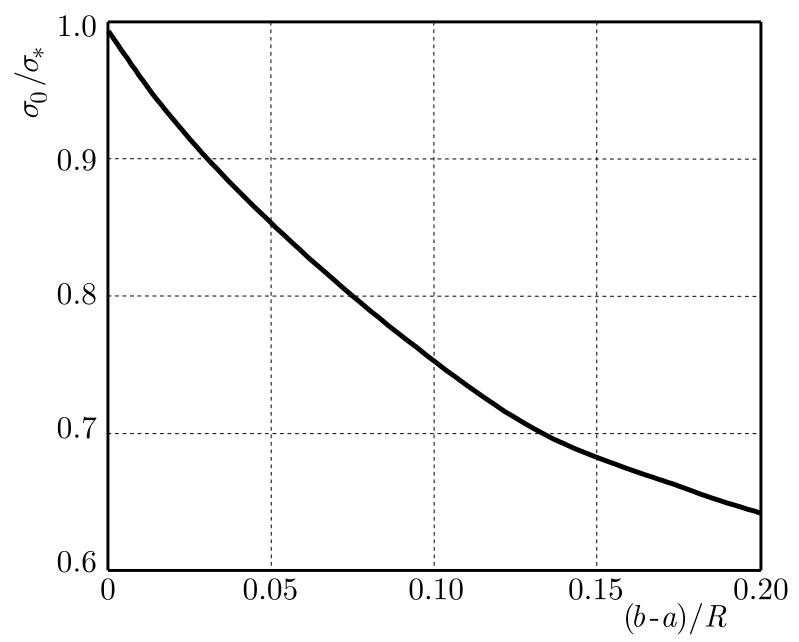

Fig. 4. Distribution of the critical load on the dimensionless length of the crack

Theoretical and experimental investigations show that the created temperature field in the course of some limited time for the purpose of crack retardation is an insurmountable barrier (Finkel, 1977) on the path of its propagation. Subsequent removal of the temperature field $(t \rightarrow \infty)$ will gradually decrease the value of compressive stresses and crack retardation efficiency. The crack faces opening at the bottom of the end zone, having attained reduction, will gradually grow to the size stipulated by mechanical load.

Under action of the temperature field, simultaneously with the reduction of maximum tensile stress, there happens its unfolding towards the thermal source. This reduces (Morozov, 1969; Finkel, 1977) the displacement of the fracture plane observed in the experiment. After removing the temperature field, this circumstance will help ensuring that for crack propagation, an increase of the external tensile load is necessary. Note that the perturbed temperature field amplifies the inhibitory effect of the induced temperature field of stresses. In conclusion, note that platelike elements have found wide application in constructions of different kind transport systems (aircraft). Based on experimental data and numerical results of this paper, we can recommend the following schemes of effective retardation of crack propagation:

- On the path of possible fracture of a plate-like construction, it is necessary to create stable temperature fields. If a crack grows in the direction of temperature increase, then velocity of its growth will decrease, and sooner or later it will stop. 
- Creating no temperature fields beforehand, heating on the path of crack propagation may be conducted impulsively, for example, by means of explosive wires. In this case, the crack tip is found in the site of explosion. As a result of simultaneous action of impact waves of thermoelastic stress and plastic deformations of the heated material, the crack growth slows down and the fracture stops.

\section{Conclusions}

Theoretical investigation of the retardation problem for a curvilinear crack with interfacial bonds by temperature fields has been carried out. An effective calculation scheme of the retardation of the curvilinear crack with interfacial bonds in a plane under action of external tensile loads is suggested. Based on the obtained results, we can consider that the temperature field created in the vicinity of the crack tip is a barrier to its propagation way. Relations for tractions in the bonds and opening of curvilinear crack faces in the end zone depending on the applied load, intensity of thermal source, crack length, and geometrical sizes of the heated zone are obtained. The dependence of the crack length on the applied stretchable load, intensity of the heated zone and also on physical and geometrical parameters of the plate at monotone loading is established.

In the case of a crack with bonds in the end zones and temperature stresses induced by heat sources, the analysis of the limiting equilibrium state of the plane reduces to a parametric study of the solution of algebraic systems (4.2),(4.3) and (4.9), (4.10) for various laws of deformation of the bonds, sizes of end zones and thermal and elastic constants of the plane material. The normal and tangential stresses in the bonds and the crack opening are directly determined by solving the resulting algebraic systems in each approximation. The crack opening in the end zones can also be determined from relation (4.11).

\section{References}

1. Barenblatt G.I., 1961, The mathematical theory for equilibrium cracks formed on brittle fracture (in Russian), Journal of Applied Mechanics and Technical Physics, 2, 4, 3-56

2. Dimaki A.V., Mel'nikov A.G., Pleshanov V.S., Sizova O.V., 2010, Theoretical and experimental study of the healing of surface cracks using induction heating, Inorganic Materials: Applied Research, 1, 4, 353-358

3. Dugdale D.S., 1960, Yielding of steel sheets containing slits, Journal of the Mechanics and Physics of Solids, 8, 100-108

4. Engineering Fracture Mechanics, The special issue: Cohesive Models, 2003, 70, 15, 1741-1987

5. Finkel V.M., 1977, Physical Basis of Fracture Retardation (in Russian), Metallurgiya, Moscow

6. Fu Y.-M., Bai X.-Z., Qiao G.-Y., Hu Y.-D., LuAN J.-Y., 2001, Technique for producing crack arrest by electromagnetic heating, Materials Science and Technology, 17, 1653-1656

7. Georgantzinos S.K., Anifantis N.K., 2014, Crack closure, [In:] Encyclopedia of Thermal Stresses, R.B. Hetnarski (Edit.), Springer Netherlands, ISBN 978-94-007-2738-0, 774-779

8. Il'yushin A.A., 2003, Plasticity (in Russian), Logos, Moscow

9. Iтоu S., 2014, Thermal stresses around two upper cracks placed symmetrically about a lower crack in an infinite orthotropic plane under uniform heat flux, Journal of Theoretical and Applied Mechanics, 52, 617-628

10. Kadiev R.I., Mirsalimov V.M., 2001, Effect of heat source on the dynamics of crack growth (in Russian), Vestnik Dagestanskogo Universiteta, 4, 69-73

11. Ladopoulos E.G., 2000, Singular Integral Equations, Linear and Non-linear Theory and its Applications in Science and Engineering, Springer Verlag, Berlin 
12. Ladopoulos E.G., 2013, Non-linear singular integro-differential equations in Banach spaces by collocation evaluation methods, Universal Journal of Integral Equations, 1, 28-38

13. Leonov M.Ya., Panasyuk V.V., 1959, Propagation of fine cracks in solid body (in Russian), Prikladnaya Mekhanika, 5, 391-401

14. LiU T.J.C., 2008, Thermo-electro-structural coupled analyses of crack arrest by Joule heating, Theoretical and Applied Fracture Mechanics, 49, 171-184

15. LiU T.J.C., 2011a, Finite element modeling of melting crack tip under thermo-electric Joule heating, Engineering Fracture Mechanics, 78, 666-684

16. LiU T.J.C., 2011b, Fracture mechanics of steel plate under Joule heating analyzed by energy density criterion, Theoretical and Applied Fracture Mechanics, 56, 154-161

17. LiU T.J.C., 2014a, Compressive stresses near crack tip induced by thermo-electric field, International Journal of Mechanical, Aerospace, Industrial, Mechatronic and Manufacturing Engineering, 8, 11, 1799-1802

18. LiU T.J.C., 2014b, Crack detection/arrest with Joule heating, [In:] Encyclopedia of Thermal Stresses, R.B. Hetnarski (Edit.), Springer Netherlands, ISBN 978-94-007-2738-0, 779-791

19. Liu T.J.C., Tseng J.F., Chen P.H., 2015, Analysis of thermo-electric field in steel strip with multiple cracks, Proceedings of the 3rd International Conference on Industrial Application Engineering, 408-412

20. Mirsalimov V.M., 1987, Non-one Dimensional Elastoplastic Problems (in Russian), Nauka, Moscow

21. Mirsalimov V.M., 2007, The solution of a problem in contact fracture mechanics on the nucleation and development of a bridged crack in the hub of a friction pair, Journal of Applied Mathematics and Mechanics, 71, 120-136

22. Mirsalimov V.M., Kadiev R.I., 2004, Closing of a crack in the sheet element under action of local thermal field, Journal of Machinery Manufacture and Reliability, 33, 6, 69-75

23. Mirsalimov V.M., Mustafayev A.B., 2015a, A contact problem on partial interaction of faces of a variable thickness slot under the influence of temperature field, Mechanika, 21, 19-22

24. Mirsalimov V.M., Mustafayev A.B., 2015b, Solution of the problem of partial contact between the faces of a slot of variable width under the action of temperature fields, Materials Science, 51, 96-103

25. Mirsalimov V.M., Mustafayev A.B., 2016, Influence of local temperature field on propagation of a curvilinear crack with interfacial bonds, ZAMM - Journal of Applied Mathematics and Mechanics, 96, 1339-1346

26. Morozov E.M., 1969, On the strength analysis by the stage of fracture (in Russian), Deform. Razrush. Term. Mekh. Vozdeistv, 3, 87-90

27. Muskhelishvili N.I., 2010, Some Basic Problem of Mathematical Theory of Elasticity, Springer, Amsterdam

28. Panasyuk V.V, Savruk M.P., Datsyshyn A.P., 1976, The Stress Distribution Around Cracks in Plates and Shells (in Russian), Naukova Dumka, Kiev

29. PARKus H., 1959, Instationäre Wärmespannungen, Springer-Verlag, Wien

30. Parton V.Z., Morozov E.M., 1985, Elastic-Plastic Fracture Mechanics (in Russian), Nauka, Moscow

31. Potthast B., Herrmann K.P., 2000, Asymptotic analysis for temperature fields induced by dynamic crack growth in pressure-sensitive materials, International Journal of Fracture, 106, $57-64$

32. Qin Z, Librescu L., Hasanyan D., 2007, Joule heating and its implications on crack detection/arrest in electrically conductive circular cylindrical shells, Journal of Thermal Stresses, 30, $623-637$ 